\title{
Grid-Forming Control for Solar PV Systems with Power Reserves
}

\author{
Bandopant Pawar, Student Member, IEEE, Efstratios I. Batzelis, Senior Member, IEEE, \\ Saikat Chakrabarti, Senior Member, IEEE, and Bikash C. Pal, Fellow, IEEE
}

\begin{abstract}
This paper presents a grid-forming control (GFC) scheme for two-stage photovoltaic (PV) systems that maintains power reserves by operating below the maximum power point (MPP). The PV plant in GFC mode behaves like a voltage source that supports the grid during disturbances in full or limited gridforming mode as per the reserve availability. This is a model-free method that avoids the estimation of MPP power in real-time commonly done in the literature, which makes it simpler and more reliable. The proposed control also features an enhanced current limitation scheme that guarantees containment of the current overshoots during faults, which is not trivial in voltage-sourced GFC inverters. A thorough investigation is done, exploring various generation mixtures of synchronous machines (SM), GFC and grid-following (GFL) inverters, and all common disturbances, e.g., load change, faults and irradiance transients. The results show very favorable dynamic performance by the GFC inverters, far superior to GFL inverters and directly comparable to SMs. It is found that replacing SMs with GFC inverters may improve the frequency profile and terminal voltage during disturbances, despite losing out in the mechanical inertia and the strict inverter overcurrent limits.
\end{abstract}

Index Terms - Ancillary services, current limitation, grid forming control, grid support, inverter, maximum power point, power reserves, renewables integration, solar photovoltaic (PV).

\section{NOMENCLATURE}

$\begin{array}{ll}\text { EMT } & \text { Electromagnetic transient } \\ \text { GF } & \text { Grid forming } \\ \text { GFC } & \text { Grid forming control } \\ \text { GFL } & \text { Grid following } \\ \text { IBRs } & \text { Inverter-based resources } \\ \text { LGF } & \text { Limited grid forming } \\ \text { MPP } & \text { Maximum power point } \\ \text { MPPT } & \text { Maximum power point tracking } \\ \text { PCC } & \text { Point of common coupling } \\ \text { PI } & \text { Proportional-integral } \\ \text { PLL } & \text { Phase-locked loop } \\ \text { PSS } & \text { Power system stabilizer } \\ \text { PV } & \text { Photovoltaic } \\ \text { PWM } & \text { Pulse width modulation } \\ \text { P\&O } & \text { Perturb and observe }\end{array}$

This work was supported in part by the Department of Science and Technology, Government of India, under the grant DST/EE/2017092 at Indian Institute of Technology Kanpur and UKSRC and the Royal Academy of Engineering under the Engineering for Development Research Fellowship scheme (no. RFI201819\18186).

(Corresponding author: Bandopant Pawar)

Bandopant Pawar and Saikat Chakrabarti are with the Department of Electrical Engineering, Indian Institute of Technology Kanpur, Kanpur, Uttar Pradesh, 208016, India (e-mail: pawarbb@iitk.ac.in; saikatc@iitk.ac.in).
RESs Renewable energy sources

RoCoF Rate of change of frequency

SM Synchronous machine

UPS Uninterruptible power source

VOC Virtual oscillator-based control

VSC Voltage source converter

VSM Virtual synchronous machine

\section{INTRODUCTION}

Pow OWER system stability concerns are growing with the increasing share of renewable energy sources (RESs) [1], especially variable and inverter-based resources (IBRs) like photovoltaic (PV) and wind generation. This has motivated the evolution of power electronic converters to provide gridsupport services ([1], [2]) and allow for more RESs integration into the power system. The majority of grid codes now request grid support and ancillary services from RESs [3].

Traditionally, the power converters of IBRs have been operating in grid-following (GFL) mode, acting essentially as current sources in the network. A range of ancillary services may be available by GFL inverters, such as steady state voltage support through reactive power injection, dynamic voltage support, fault ride through, and primary frequency support in terms of droop and inertial response [1]-[4]. Such support by GFL converters may be not very effective during a disturbance for several reasons ([1], [5]-[7]): i) sensing and actuation delays, ii) weak grids due to decreasing synchronous generation and remote IBR connection, and iii) challenges in the phase locked loop (PLL) and rate of change of frequency (RoCoF) measurement. These limitations impede high GFL-based RESs penetration to the grid [8], [9].

A promising alternative is to operate an IBR in grid-forming control (GFC) mode [1], [10]-[11]. In contrast to GFL, the GFC converter acts as a controllable voltage source that can establish voltage and frequency in the network. The GFC source is robustly synchronized to the grid, and any disturbance is automatically catered instantly, not relying on a PLL that malfunctions in weak grids like in the GFL case [8]. Stabilitywise, GFC exhibits superior dynamic performance over GFL

Efstratios I. Batzelis is with the School of Electronics and Computer Science, University of Southampton, Southampton, SO17 1BJ, UK (e-mail: e.batzelis@soton.ac.uk).

Bikash C. Pal is with the Department of Electrical and Electronic Engineering, Imperial College, London SW7 2AZ, U.K. (e-mail: b.pal@imperial.ac.uk). 
and synchronous machines (SMs) in terms of frequency stability [12]-[13] and short-term voltage stability [14]. In addition, GFC is necessary for islanded operation and black start services, functions that will be critical in a high-IBR future power system [8]. GFC is the only way for a power system with $100 \%$ IBRs and a promising method for high-RESs penetration [10]-[11].

Although GFC has been used for years in standalone systems, such as isolated microgrids and uninterruptible power supply (UPS) [15], its scope has been extended recently to the entire power system [16]. There are a few variants of GFC implementation depending on how the reference voltage is generated [12],[16], such as droop control [12]-[17], virtual synchronous machine (VSM) control [13],[17-18], matching control [13],[17],[19], and virtual oscillator based control (VOC) [13],[20]. All these alternatives can be tuned for a similar steady-state response, but they differ during transient conditions as per their characteristics [13], [8]. Out of these, droop control is the most mature and widely used [8].

The majority of studies in the literature on GFC assumes an ideal source or battery on the input side [12]-[20]. However, the primary source of RESs, such as PV plants, is stochastic and non-dispatchable and should not be treated as such. There are mainly two methods of providing ancillary services from PV plants: either by hybridizing with energy storage, or by maintaining reserves with a curtailed operation (below the maximum power point (MPP)) ([2], [8], [21]-[25], [26]). The former option provides maximum flexibility (e.g., nigh-time operation), but comes with additional installation and maintenance costs. The curtailed (or deloaded or power reserves or power headroom) operation under-utilizes the installed PV capacity and is only available during the day-time, but it is simpler and does not involve additional equipment and costs ([2], [26]). With the increasing share of renewable sources into the grid, there is a growing consensus for utilization of large-size utility-scale PV plants to provide grid reliability services in the grid-forming mode without using energy storage ([8], [21]-[25]). This is the focus of this paper.

A big challenge in this approach is monitoring the maximum power during curtailed operation as it changes with time. Estimation-based approaches for the PV grid-forming operation has been explored in [21]-[25] to monitor the MPP at deloaded conditions. In [21], a curtailed PV generator is used as an input source assuming real-time MPP estimation, but no detailed model is given. [22]-[25] focus on maintaining the power reserves and not on grid support during disturbances. [21],[22],[24] have tested the GFC strategy only for load change disturbance, while [25] for voltage sags; none of them have tested the control performance for all types of common disturbances such as load change, irradiance change, and especially network faults.

Furthermore, real-time MPP estimation adopted in all aforementioned studies comes with many challenges, such as parameter uncertainty, estimation accuracy, poor performance during a sudden change in irradiance, etc., [27]-[28]. For example, abrupt changes in irradiance may occur due to cloud movement, with recorded transients of up to $150-200 \mathrm{Wm}^{-2} \mathrm{~s}^{-1}$ in some cases [26],[29]. For this reason, the GFC proposed in this paper is model-free and does not employ any MPP estimation.

Furthermore, all available GFC studies for PV plants assume single-stage systems, i.e., there is only a single DC/AC conversion stage [22]-[25]. However, two-stage PV systems comprising both a DC-DC converter and an inverter are increasingly more common in the network. They are also better candidates for GFC due to a wider operational range from nearzero to $100 \%$ of the available PV power [30]; this contrasts with the single-stage systems that struggle to operate at very low power outputs due to DC link voltage limitations. This paper aims to cover this gap by proposing a GFC control scheme designed for two-stage PV systems that is missing from the literature.

Another important consideration in GFC is the limited overcurrent capacity of power converters, which, if exceeded during transients, may result in catastrophic failure of the power switches. Adhering to this limitation is particularly challenging in GFC, due to the voltage-source nature of the converter as opposed to the current-source-behaved GFL. Available current limitation methods in the literature involve either virtual impedance schemes or reference current saturation [31]-[33]. The former approach emulates the effect of a virtual impedance connected at the output of the inverter, whose value is adjusted during overloads to contain the current overshoots. This method, however, may struggle to limit the current during the first few cycles and may result in oscillatory behavior [32], [33]. In the reference current saturation scheme, the voltage controllers' output, i.e., the reference current, is saturated to contain the overcurrent, usually employing an anti-wind-up mechanism. A relevant implementation is studied in the MIGRATE project [31], concluding that during the saturation the controller input remains unregulated and some initial current overshoots are still apparent [31],[32]. In MIGRATE, a hybrid combination of the two aforementioned approaches is proposed [31], which however seems too complex to parametrize and tune in large systems. This paper presents a modified reference current saturation strategy that successfully contains the initial overcurrent and guarantees a non-oscillatory response. Highlights of the proposed technique are an antiwind-up scheme that accounts for the feedforward signals, rate limiters at the controller's outputs and adjustable saturation bounds.

This paper proposes, for the first time, a model-free GFC strategy with a current limitation mechanism for two-stage PV systems with power reserves. The key contribution points of this research are:

- A model-free method that does not need to estimate the MPP, in contrast to the available literature

- Designed for two-stage PV systems without energy storage for the first time

- Utilizing the limited reserves optimally by operating in two modes: full and limited grid-forming

- A new current limitation scheme based on a modified reference current saturation that contains overshoots 


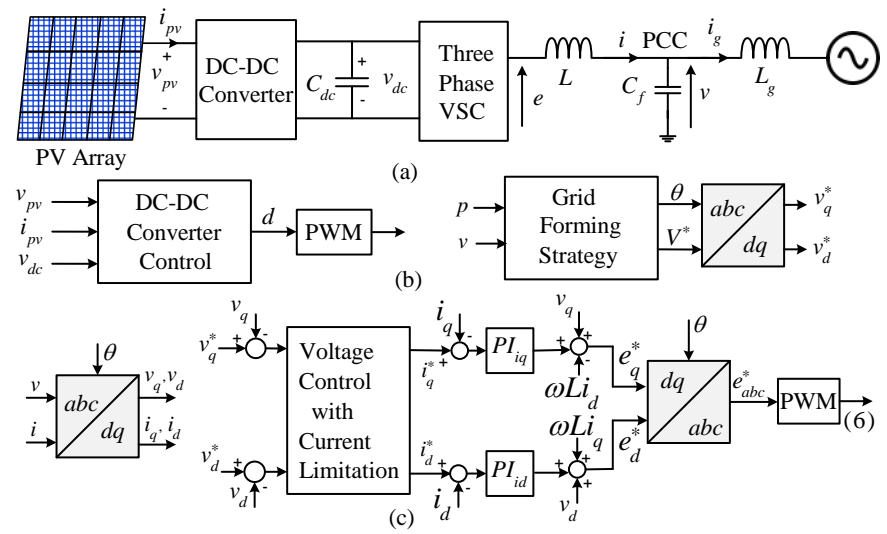

Fig. 1. The overall GFC scheme for a two-stage grid-connected PV plant, (a) PV system topology, (b) DC-DC converter control and outer inverter control loop, (c) inner inverter control loops with current limitation scheme.

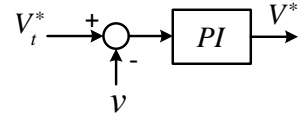

(a)

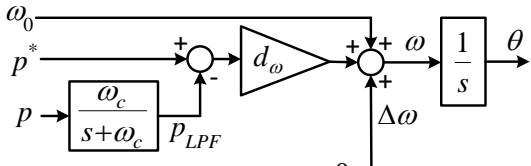

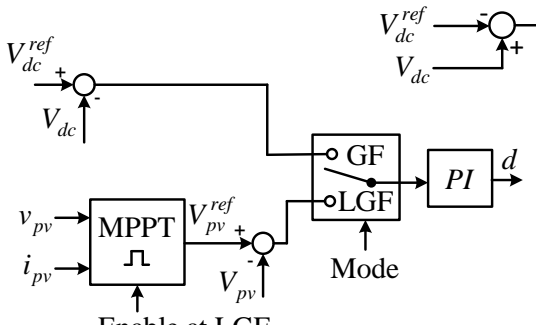

(c)
Fig. 2. GFC strategy to generate the (a) inverter reference voltage, (b) inverter reference frequency, (c) DC-DC converter duty ratio.

- Validating the new control at all common major disturbances (load, irradiance, faults), usually missing from relevant studies

- Validation at various generation mixtures of machines, GFC and GFL inverters at the WSCC 9-bus testbench.

The remainder of the paper is organized as follows. In Section II, the proposed control scheme is described in detail. Section III covers the controller tuning method, Section IV focuses on modeling details of the simulated system, followed by the results in MATLAB/Simulink in Section V. Section VI concludes the paper.

\section{Proposed Grid-Forming CONTROL SCHEME}

The GFC inverter acts as a controllable voltage source in the network, whose power output is determined by the loading conditions in the grid, rather than the primary source availability like in GFL inverters. This entails that during disturbances, GFC resources will remain strongly coupled to the grid and inherently supply the required active and reactive power. This contrasts with GFL inverters that need to sense the disturbance and employ additional dedicated control loops for grid support, such as frequency response or dynamic voltage support.

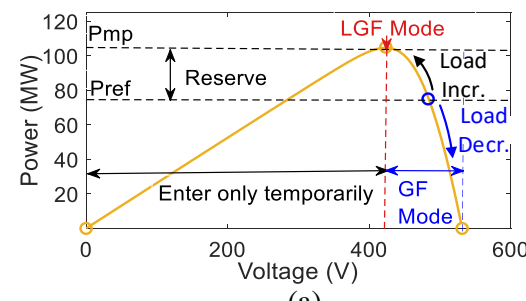

(a)

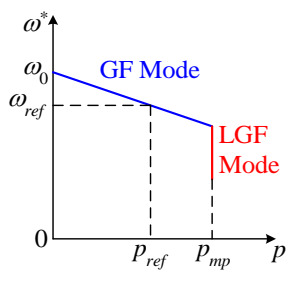

(b)
Fig. 3. (a) The P-V characteristic of the PV plant and different operating regions, (b) Power-frequency characteristics of the VSC.

\section{A. Overall Control Scheme}

The overall GFC scheme for a two-stage grid-connected PV system is shown in Fig. 1. Fig. 1(a) depicts the system topology. Fig. 1(b) shows the DC-DC Converter Control and the outer inverter control loop (Grid Forming Strategy block) that generates the reference voltage and frequency to be tracked by the inner inverter loops in Fig. 1 (c). Fig. 1(c) involves the voltage control loop featuring a current limitation mechanism and the inner current control loop that produces the inverter modulation signals fed into a PWM generator.

In the conventional GFL mode, the DC-DC converter determines the extracted power from the PV array, and the inverter follows by transferring that power to the grid. However, in GFC, these roles are reversed: the inverter adjusts its output autonomously based on the grid conditions, while the DC-DC converter follows by matching that power demand.

It is worth noting that a PLL is not required during the normal operation of a grid-forming inverter. It may be used, however, temporarily for synchronization purposes when the system is first connected to the grid [34]-[35], [8], deactivated thereafter during normal operation.

\section{B. Grid Forming Strategy and DC-DC Converter Control}

The proposed GFC strategy has two modes of operation: full grid-forming (GF) when the inverter output is within the capacity of the PV array and limited grid-forming (LGF) when it exceeds the maximum available PV power. In GF mode, the inverter feeds in power based on a droop equation, and the DCDC converter regulates the dc-link; in LGF mode, the inverter performs the dc-link regulation instead, while the DC-DC converter reverts to maximum power point tracking (MPPT) operation.

Fig. 2 illustrates the complete control diagram, some parts being active only in one of the two modes. Fig. 2(a) shows a simple closed-loop voltage control that regulates the terminal voltage $v=\sqrt{v_{d}^{2}+v_{d}^{2}}$ to the reference $V_{t}^{*}$ and determines the inverter reference voltage $V^{*}$. Fig. 2(b) depicts the powerfrequency $(p-f)$ droop scheme that generates the inverter frequency $\omega$ and angle $\theta$ based on a modified droop equation:

$$
\omega=\omega_{0}+d_{\omega}\left(p^{*}-p_{L P F}\right)+\Delta \omega
$$

where $\omega_{0}$ is the nominal frequency, $d_{\omega}$ the droop coefficient, $p^{*}$ the power setpoint and $p_{L P F}$ the low-pass filtered measurement of the output power $p$ ( $\omega_{c}$ is the cutoff frequency). $\Delta \omega$ is a frequency adjustment produced by a PI controller that regulates the dc-link voltage $V_{d c}$ to its reference $V_{d c}{ }^{r e f}$ and is active only in LGF mode. The purpose of this additional loop is to bring the power output back within the PV capacity in LGF mode. 


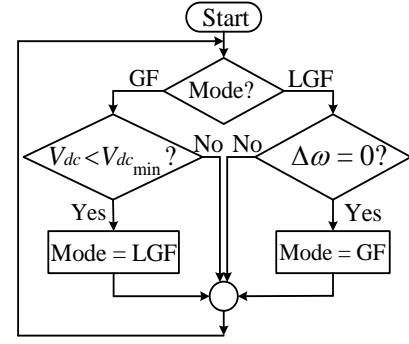

(a)

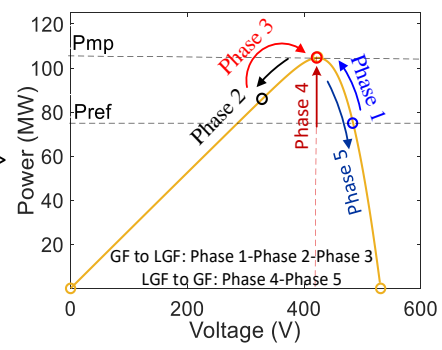

(b)
Fig. 4. The change in the mode of operation: (a) flowchart, (b) example mode transitions.

The DC-DC converter control shown in Fig. 2(c) comprises two branches, only one being active at a time, depending on the mode. In normal GF mode, a PI controller regulates the dc-link voltage; in LGF mode, an MPPT algorithm is executed instead to extract the maximum available power from the PV array.

\section{1) Full grid-forming $(G F)$ mode}

To allow for both up- and down-regulation of its power output depending on the disturbance, the PV system operates in a deloaded manner (i.e., curtails power, keeps power reserves, maintains a power headroom, dispatchable operation), as shown in Fig. 3(a). The power setpoint and reserve power will be decided by the power system operator according to the forecasts-based scheduling and encouraging market mechanism for grid support from renewable sources. The power setpoint is an input coming from the system operator in the proposed control scheme. For any setpoint $p^{*}$ below the MPP, there are two possible operating points on either side of the P-V curve. Here the operating point is kept in the right-hand side of the MPP, which allows operation at the entire power range $(0$ $100 \%$ of the MPP power) and results in higher PV voltage and DC-side efficiency [30].

Given a disturbance, e.g., a load decrement, the output power drawn from the inverter will lower, thus mobilizing the droop control (Fig. 2(b)) to adjust the frequency $\omega$ according to the characteristic of Fig. 3(b), and the DC-DC converter control (upper branch in Fig. 2(c)) to shift the operating point towards the right (blue arrow in Fig. 3(a)). If the load increases, the controller responds in the opposite direction, shifting the operating point to the left (black arrow in Fig. 3(a)).

\section{2) Limited grid-forming ( $L G F)$ mode}

If the disturbance is too large, it may be possible that the operating point overtakes the MPP and lands on the left-hand side of the curve. If no action is taken, the DC-DC converter will drive the operating point further to the left by increasing the duty ratio trying to meet the power target, which will lead to instability. For this reason, the control is switched to LGF mode once the operating point overtakes the MPP.

In LGF mode, the DC-DC converter switches to MPPT operation (lower branch in Fig. 2(c)), and the frequency adjustment branch in the droop scheme is activated (lower branch in Fig. 2(b)). By reducing the frequency $\omega$, the inverter output is brought back within capacity, i.e., at maximum power minus losses. During this mode, the inverter can still support down-regulation of the output but not up-regulation, and thus it is denoted as limited grid-forming.

It is worth highlighting that with this approach, a conventional model-free MPP tracking algorithm is employed
(Perturb and Observe algorithm), which is simple and agnostic of the system structure and parameters [36]. This is in contrast to existing alternatives that adopt real-time MPP estimation methods [24], [26], [30] that are based on mathematical models and are prone to accuracy and convergence issues.

\section{Change in Mode of Operation}

The mode transition from GF to LGF and vice versa is illustrated in the flowchart of Fig. 4(a), explained below in detail using the examples in Fig. 4(b).

\section{1) Mode transition from $G F$ to $L G F$}

Under normal conditions in GF mode, the setpoint $p^{*}$ in Fig. 4(b) is met by the system, $\Delta \omega$ in Fig. 2(b) is zero, and the dclink is regulated by the DC-DC converter. If a disturbance causes a power deficit (e.g., load increase, generation outage, or irradiance drop), the dc-link voltage will start dropping, mobilising the DC-DC converter to increase the duty cycle and shift the operating point to the left (Phase 1 in Fig 4(b)). As long as the new equilibrium lies in the right-hand-side of the MPP, operation continues in GF mode as normal.

However, if the disturbance is so large that the power balance cannot be met, the linear action of the controller will drive the operating point to the left side, overtaking the MPP (Phase 2). This will further increase the power gap, moving to lower voltages and risking instability if the mode is not changed to LGF. The inability to meet the power balance is reflected in the dc-link voltage, which keeps falling; when it falls below a threshold $V_{d c m i n}$, the flowchart of Fig. 4(a) changes the mode to LGF, thus reverting to MPPT operation in the DC-DC converter (Phase 3) and activating the frequency adjustment branch in the inverter control to limit the inverter power injection.

2) Mode transition from $L G F$ to $G F$

While being in LGF mode, the dc-link is managed by the inverter by means of the frequency adjustment $\Delta \omega$ and the DCDC converter tracks the MPP (Phase 4). If the drawn power from the inverter gets back within PV capacity (e.g., disturbance ends), the power surplus in the dc-link will result in increasing $\Delta \omega$ (negative value) till it gets zero. When this happens, the system is again fully capable of supporting the grid, and the mode is changed back to GF (flowchart in Fig. 4(a)). This way, the DC-DC converter undertakes the dc-link regulation once again, shifting the operating point to the righthand side (Phase 5), and everything returns to normal GF operation.

\section{Proposed Current Limitation Scheme}

The details of the voltage control with current limitation (see Fig. 1) are given in Fig. 5. It comprises two PI controllers that regulate the inverter voltages $v_{q}$ and $v_{d}$ to their reference values $v_{q}{ }^{*}$ and $v_{d}{ }^{*}$ by adjusting the active and reactive reference currents $i_{q}{ }^{*}$ and $i_{d}{ }^{*}$. They also involve the standard feedforward signals: grid currents $i_{g q}$ and $i_{g d}$, and the capacitor currents of the grid-side filter $\omega C_{f} v_{d}$ and $\omega C_{f} v_{q}$.

To avoid current overshoots during transients, the controllers' outputs are saturated within $\left[0, I_{q, \text { lim }}\right]$ for the active and $\left[-k_{l} I_{n}, k_{l} I_{n}\right]$ for the reactive current. For these limits, it is assumed that the inverter can withstand a transient current of up to $k$ times the rated value $I_{n}$ (e.g., $k=1.414$ ); this margin is deployed primarily for reactive current (reactive current priority) up to $k_{l}$ times the nominal $I_{n}$ (e.g., $k_{l}=1.2$ ), and what 


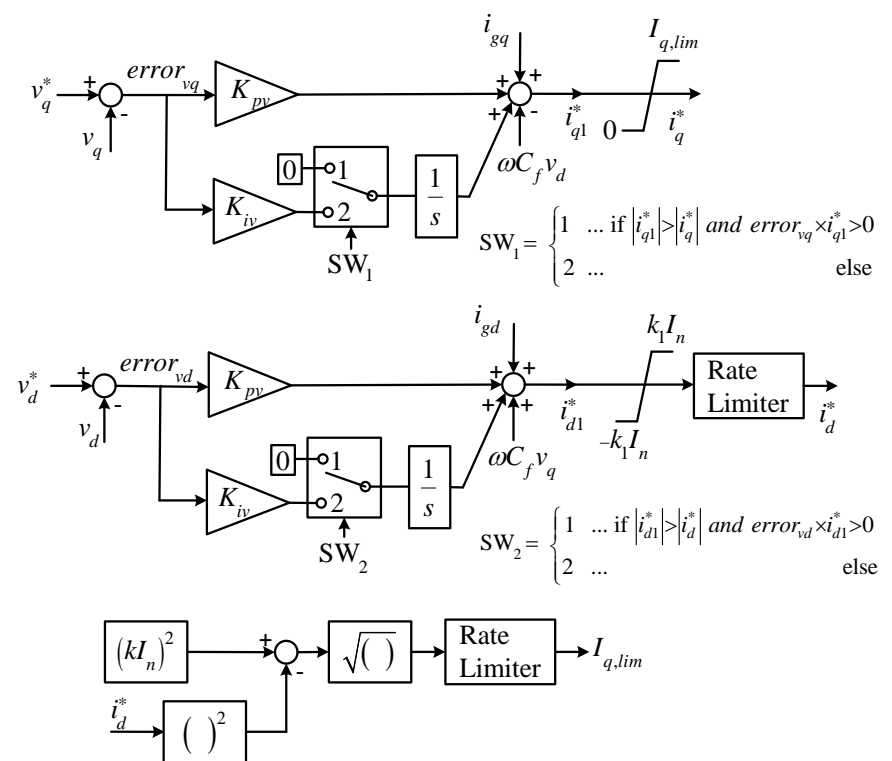

Fig. 5. The proposed current limitation scheme.

remains determines the active current limit $i_{q, \text { lim }}$ (lower branch of Fig. 5). In addition, two rate limiters at $i_{d}{ }^{*}$ and $I_{q, \text { lim }}$ do not allow very high changes and contribute to minimizing the initial current overshoot and damping any oscillations.

This scheme mitigates the main weakness of the reference current saturation scheme examined in MIGRATE project [31], i.e., initial peak current exceeding the limit at the onset of the fault. By applying adjustable saturation limits for the real and reactive current and rate limiters to damp very fast changes, the proposed scheme manages to contain the initial overshoot more effectively and avoid any undesirable oscillations.

In addition, an enhanced anti-wind-up scheme takes into full consideration the feedforward paths of the controllers to ensure smooth return to normal operation when the disturbance ends. In Fig. 5, the anti-windup method conditionally prevents error accumulation in the integrators. This is managed by the switches SW1 and SW2 that effectively freeze the integrators by feeding them a zero signal during the saturation. This happens when the input and output of the saturation blocks differ $\left(\left|i_{q}^{*}\right|<\left|i_{q 1}^{*}\right|,\left|i_{d}^{*}\right|<\left|i_{d 1}^{*}\right|\right)$ and the error signal has the same sign as the pre-saturation output (error ${ }_{v q} i_{q 1}^{*}>0$, error $_{v d} i_{d 1}^{*}>0$ ) that indicates saturation and undesirable error accumulation. This clamping anti-windup method is referred to as conditional integration in the literature, as it prevents error accumulation in one particular direction. The proposed current limitation scheme contains the overshoot reliably and autonomously, without additional functions like fault detection or control switching seen in the literature, which simplifies tuning and implementation.

\section{CONTROLLER TUNING}

This section briefly discusses the tuning and stability aspects of the controllers involved in the proposed scheme. The inner inverter control loops, i.e. voltage control loop and current control loop, and the terminal voltage control are well studied in the literature [40]. Furthermore, frequency adjustment controllers similar to the one proposed in Fig. 2(b) are available in the literature [21]-[25].

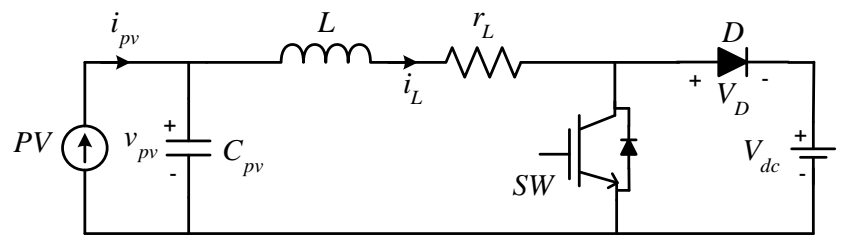

Fig. 6. Equivalent circuit of the Boost converter in LGF mode.

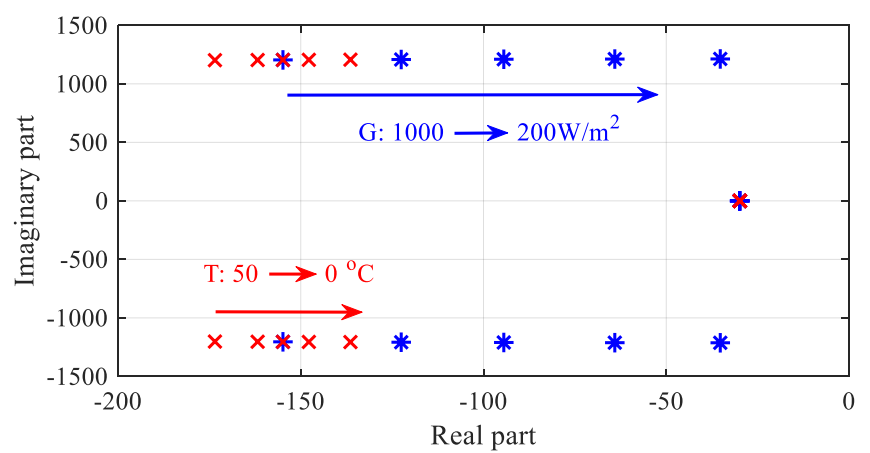

Fig. 7. Closed loop poles for the Boost converter in LGF mode with varying irradiance and temperature conditions.

Therefore, in this section we focus only on the newly introduced PI controller of the Boost converter (Fig. 2(c)). The tuned parameters of all controllers are given in the appendix.

The Boost converter control scheme of Fig. 2(c) employs the same PI controller for both full GF and LGF operation. Therefore, the controller should be tuned for both modes: the PI gains are first tuned for the LGF mode and then it is shown to have favorable operation in the GF mode as well.

In LGF mode, the boost converter operates in MPPT and the DC link is regulated by the inverter. The equivalent circuit of Fig. 6 treats the PV generator as current source linearized around the operating point and the DC link as a constant voltage source [41]. The state-space model is given by (2) and (3) [41]:

$$
\begin{gathered}
\frac{d}{d t}\left[\begin{array}{c}
\hat{i}_{L} \\
\hat{v}_{p v}
\end{array}\right]=\left[\begin{array}{cc}
\frac{-r_{L}}{L} & \frac{1}{L} \\
\frac{-1}{C_{p v}} & \frac{1}{r_{p v} C_{p v}}
\end{array}\right]\left[\begin{array}{l}
\hat{i}_{L} \\
\hat{v}_{p v}
\end{array}\right]+\left[\begin{array}{c}
\frac{\left(V_{D}+V_{d c}\right)}{L} \\
0
\end{array}\right] \hat{d} \\
\hat{y}=\left[\begin{array}{ll}
0 & 1
\end{array}\right]\left[\begin{array}{ll}
\hat{i}_{L} & \hat{v}_{p v}
\end{array}\right]^{\mathrm{T}}
\end{gathered}
$$

Here, $\hat{\imath}_{L}$ represents the incremental inductor current, $\hat{v}_{p v}$ the incremental PV voltage, $\hat{d}$ the incremental duty ratio, $L$ and $r_{L}$ the inductance and resistance of the inductor, $C_{p v}$ the PV array capacitor, $r_{p v}$ the equivalent linearized PV array resistance, $V_{D}$ the forward diode voltage, and $V_{d c}$ the nominal DC-link voltage. The PV array is modeled as a voltage-dependent current source through the PV array voltage $v_{p v}$ and the resistance $r_{p v}$. The equivalent resistance $r_{p v}$, i.e., the slope of voltage-current characteristic, changes with the PV operating point and irradiance and temperature conditions [41].

The PI controller gains tuned using Bode plots [41] yield $K_{p}$ $=0.1$ and $K_{i}=2$ values and result in infinite gain margin and phase margin of $99^{\circ}$ at STC. Fig. 7 illustrates the closed-loop poles for varying irradiance and temperature, proving that the system remains stable at all operating conditions. 


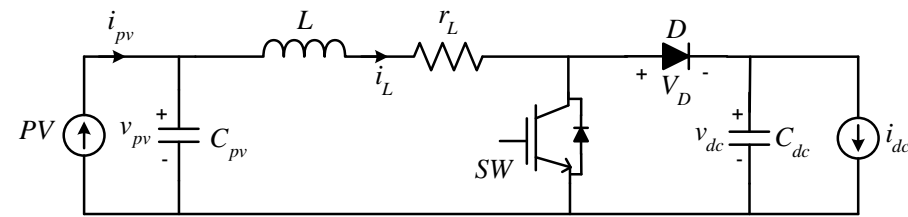

Fig. 8. Equivalent circuit of the Boost converter in GF mode.

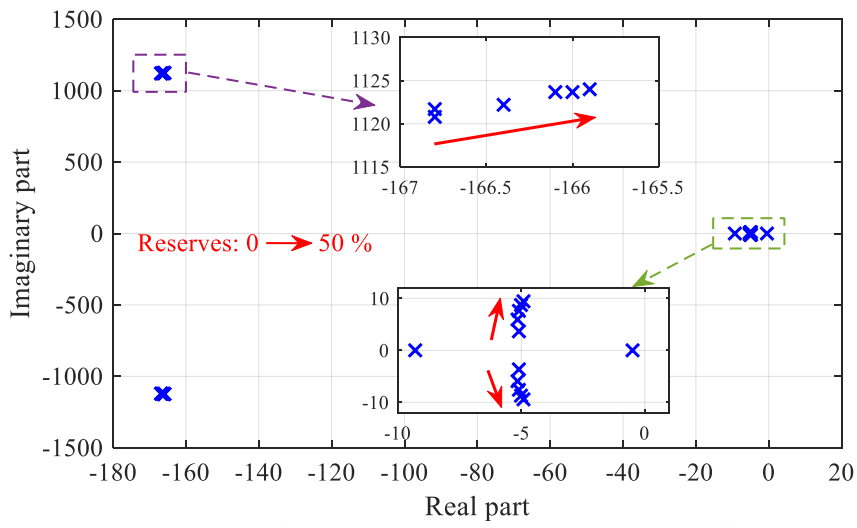

Fig. 9. Closed loop poles for the Boost converter in GF mode at different reserve levels.

In GF mode, the Boost converter regulates the DC link while the inverter transfers power independently. Extending the previous approach, the equivalent model of Fig. 8 treats now the inverter as a current source modeled through the DC-link voltage $v_{d c}$ and resistance $r_{d c}$. The resistance $r_{d c}$ changes with the operating point, i.e., the power injected by the inverter. The following state-space model involves now an additional state, i.e. the incremental DC-link voltage $\hat{v}_{d c}$, and the DC-link capacitance $C_{d c}$.

$$
\begin{gathered}
d t\left[\begin{array}{c}
\hat{i}_{L} \\
\hat{v}_{p v} \\
\hat{v}_{d c}
\end{array}\right]=\left[\begin{array}{ccc}
\frac{-r_{L}}{L} & \frac{1}{L} & \frac{-(1-D)}{L} \\
\frac{-1}{C_{p v}} & \frac{1}{r_{p v} C_{p v}} & 0 \\
\frac{(1-D)}{C_{d c}} & 0 & \frac{-1}{r_{d c} C_{d c}}
\end{array}\right]\left[\begin{array}{l}
\hat{i}_{L} \\
\hat{v}_{p v} \\
\hat{v}_{d c}
\end{array}\right]+\left[\begin{array}{c}
\left(V_{D}+V_{d c}\right) \\
0 \\
-I_{L} \\
C_{d c}
\end{array}\right] \hat{d} \\
\hat{y}=\left[\begin{array}{lll}
0 & 0 & 1
\end{array}\right]\left[\begin{array}{lll}
\hat{i}_{L} & \hat{v}_{p v} & \hat{v}_{d c}
\end{array}\right]^{\mathrm{T}}
\end{gathered}
$$

The previously selected PI controller parameters yield a gain margin of $50 \mathrm{~dB}$ and a phase margin of $57^{\circ}$ at STC and $30 \%$ reserves in GF mode. Fig. 9 depicts the closed-loop system poles at various reserve levels, indicating stability at all conditions. This investigation confirms that the selected controller gains are suitable at all conditions for both GF and LGF operating modes.

\section{TEST System}

The WSCC 9-bus testbench [37]-[38] is considered in this paper to validate the proposed control scheme for different generation mixtures. First, the original version with SMs as generators is used as a benchmark (All SM case). Then, a PV plant employing the proposed GFC strategy replaces the generator at bus 3, as shown in Fig. 10 (SM GFC case); this refers to a near-future scenario where SMs still dominate the power system, and the PV plant has to coordinate with the slow-

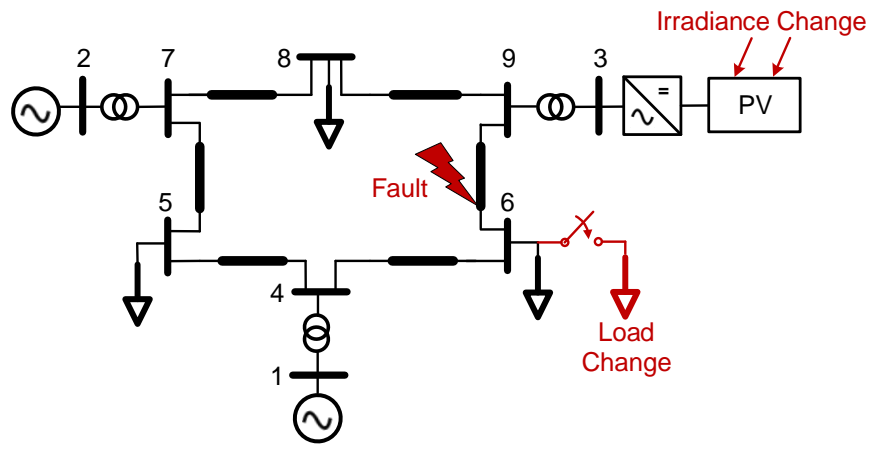

Fig. 10. Modified WSCC 9-bus test system used in the case studies.

acting machines to avoid overloading, thus limiting its full potential. Thirdly, all generators are replaced by GFC inverters (All GFC case), with a PV array as input at bus 3 and ideal sources elsewhere; this represents a future inverter-dominated power system, where fast-acting inverters can deploy their full potential. Furthermore, to assess and compare the performance of grid-following sources, the SM GFC and All GFC cases are then modified to replace some generators with inverters running in GFL mode with grid-support capability. The respective SM GFL and GFC GFL cases are examined to explore the limitations of today's grid-following approach for IBRs. The system dynamics are examined in these cases at various disturbances, namely, load change, irradiance transient, and line fault, as shown in Fig. 10.

The simulations are carried out in electromagnetic transient (EMT) mode in MATLAB/Simulink. The detailed seventh order model of the SM is used, employing IEEE type DC1A exciter and voltage regulator with a two lead-lag stage power system stabilizer (PSS). Usually, the droop coefficient in SMs varies between $2 \%$ and $5 \%$; here, the minimum value of $2 \%$ with the standard TGOV1 governor model is considered. The inverter droop coefficient can be much lower, here assumed at $1 \%$. The transmission lines are modeled by the distributed parameter model and loads by constant impedances. The parameters of the test system are taken from [37]-[38].

The PV plant of $105 \mathrm{MW}$ nominal capacity is scheduled at $75 \mathrm{MW}$ prior to the disturbance, keeping $30 \mathrm{MW}$ of reserves. An aggregate model for the PV plant is used here to reduce the computational burden, as commonly done in system-level studies. The PV park is represented by a structure-preserving aggregate model of the VSC [39], extended to the DC-DC converter as well. A $25 \mathrm{kVA}$ unit that includes a DC-DC converter and an inverter is used as building block to connect several such units in parallel for the required plant size. The AC output voltage across the filter capacitor is $400 \mathrm{~V}$ and the nominal DC-link capacitor voltage is $750 \mathrm{~V}$. The inverter output voltage is stepped up to $11 \mathrm{kV}$ and further to $230 \mathrm{kV}$ using the step-up transformers. It is worth noting that the proposed control strategy works perfectly fine with other converter sizes and voltage levels, as long as it is a two-stage system. The simulations are based on the average-value converter models, as commonly done in dynamic simulations of power system studies. The parameters of the PV system are given in the appendix. 

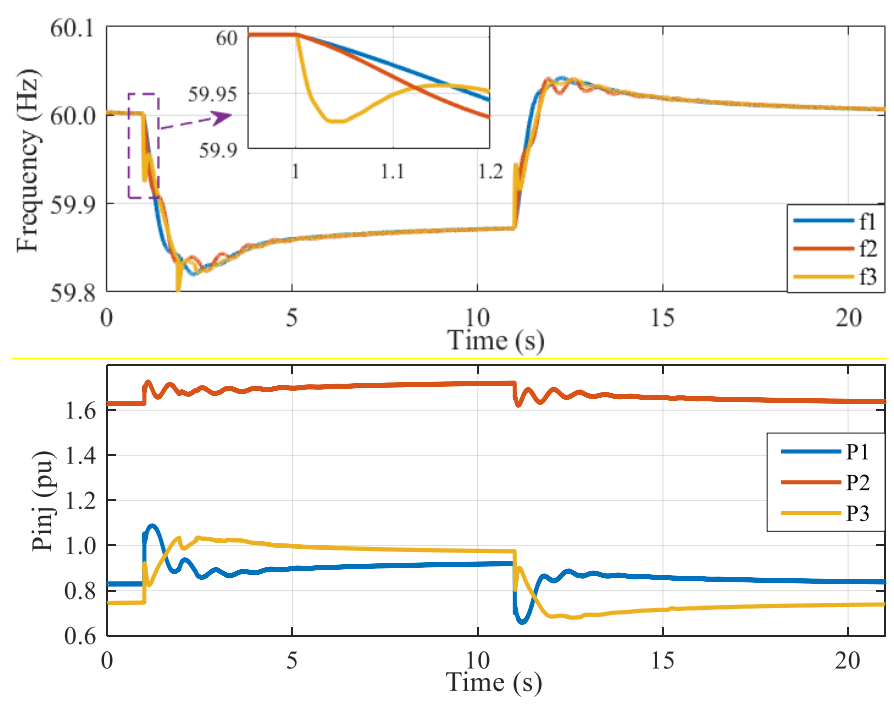

Fig. 11. Frequency and active power injection in the SM GFC case subject to a load change at bus 6 .
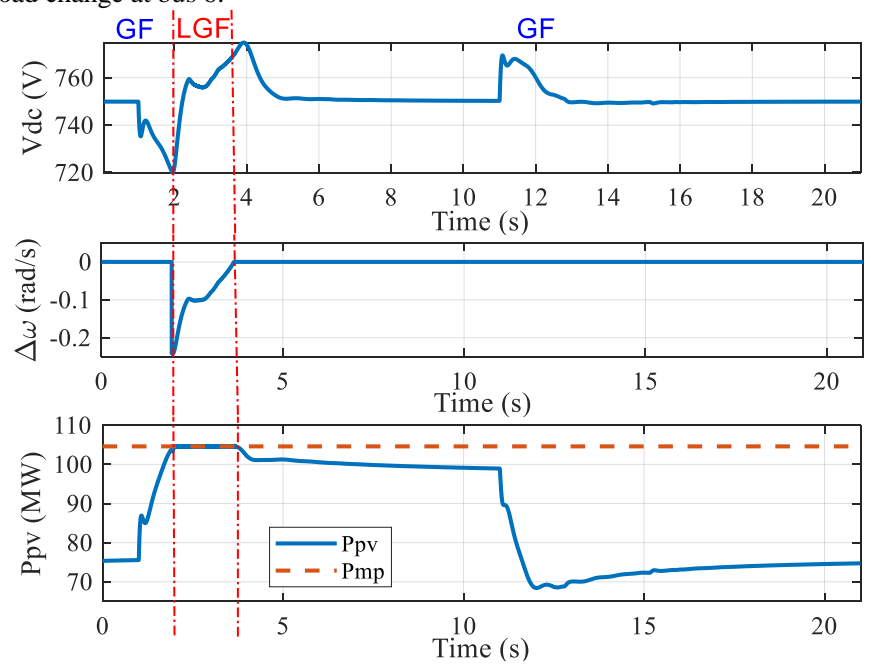

Fig. 12. Performance of the proposed control strategy in the SM GFC case subject to a load change at bus 6 .

\section{Simulation Results}

\section{A. SM GFC case (two SMs and one PV GFC plant)}

In this scenario, it is examined how the PV GFC system can support the grid during load changes and faults, as well as what the impact of the varying PV generation on the system is.

\section{1) Load Disturbance}

A load of $47.25 \mathrm{MW}$ (15\% of total system load) is switched $\mathrm{ON}$ at $\mathrm{t}=1 \mathrm{~s}$ and switched off at $\mathrm{t}=11 \mathrm{~s}$ at bus 6 as shown in Fig. 10. The system dynamics are given in Fig. 11. The PV GFC (yellow lines) exhibits a similar response to that of the SMs, except that it shares a larger portion of the load due to lower droop settings. Furthermore, the near-zero time-constants of PV GFC entail faster response and power injection to the disturbance compared to the SMs, which results in more rapid drop of the PV GFC frequency $f_{3}$ in Fig. 11 at the first few cycles. Still, as the system remains in synchronism, the power imbalance is met quickly this way and the nadir of the common frequency in the network is higher than what it would be with All SM.

The performance of the proposed control strategy is given in Fig. 12. The additional power drawn from the inverter results in
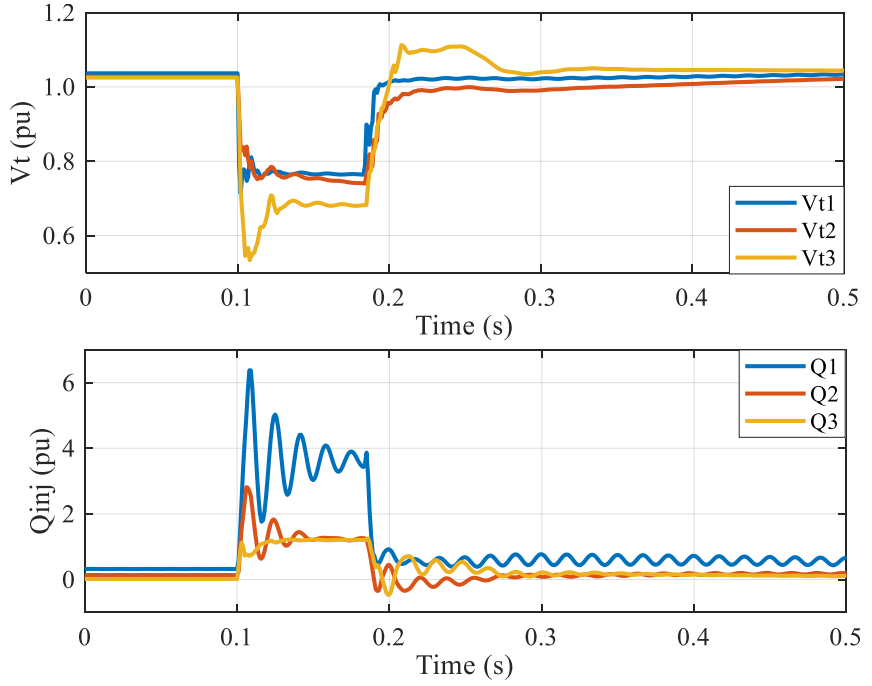

Fig. 13. Terminal voltage and reactive power injection in the SM GFC case subject to a three-phase fault.

PV power increase by deploying the maintained reserves up to the MPP. Still, this is not enough, with the operating point overtaking the MPP and the dc-link voltage dropping till the threshold $720 \mathrm{~V}\left(V_{d c}\right.$ plot in Fig. 12) that triggers switching to LGF mode (see annotations in Fig. 12). In LGF mode, the $\Delta \omega$ frequency adjustment contains the inverter output power while extracting maximum power from the PV array (Delta- $w$ and $P p v$ plot in Fig. 12), which allows the dc-link voltage to return back to normal. Since this is a transient overload condition and the steady-state inverter output is within the PV capacity, $\Delta \omega$ is gradually zeroed, and the mode switches back to GF at about $\mathrm{t}$ $=3.6 \mathrm{~s}$ (Fig 12). When the load is switched OFF at $\mathrm{t}=11 \mathrm{sec}$, the control works smoothly in the opposite direction as well: the inverter reduces its output and brings the operating point back at the initial levels.

It is worth noting that with this approach, the power imbalance and mode change is perceived by the voltage drop in the DC link. This inevitably entails a short delay in the response in the order of milliseconds and requires a sufficient voltage range for this sag (here $30 \mathrm{~V}$ ). Still, these values are perfectly acceptable and guarantee detection of the power imbalance, which may fail with other MPP-estimation-based approaches.

2) Three Phase Fault and Line Opening

A three-phase fault is applied on the line 6-9 at $\mathrm{t}=0.1 \mathrm{~s}$, with a fault resistance $R_{f}=1 \Omega$ (Fig. 10). The fault is cleared after five cycles by tripping the line 6-9. The system response is given in Fig. 13 and Fig. 14. Fig. 13 shows the variation of terminal voltage and reactive power injection. When the fault occurs, the SMs inject reactive currents up to three to six times their pre-fault

values, whereas the inverter current is limited to $k=1.414$ p.u. $\left(Q_{i n j}\right.$ plot in Fig. 13) Hence, the terminal voltage of the PV plant is lower than those of the SMs ( $V_{t}$ plot in Fig. 13). The oscillations in the injected currents by the SMs are due to inherent electromechanical machine dynamics, which are not apparent in the PV inverter.

Fig. 14 shows the variation of the active and reactive components $\left(I_{\text {inv }}\right.$ plot $)$ and magnitude $\left(I_{\text {mag }}\right.$ plot $)$ of the inverter current. The real part of the current, $i_{q}$, shoots up for the initial period of around a cycle, while the reactive part of the current, 

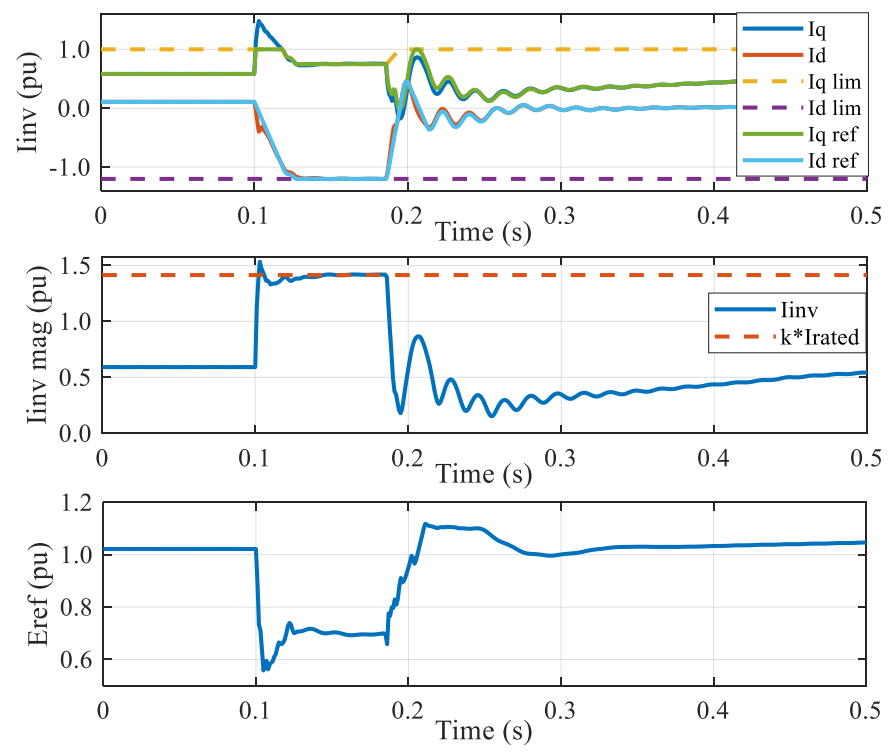

Fig. 14. $d q$ components and magnitude of the inverter current, and magnitude of the VSC reference voltage in the SM GFC case subject to a three-phase fault.

$i_{d}$, takes around a cycle to reach the limit of 1.2 p.u. due to the rate limiter. Hence, the terminal voltage dips for a period of around a cycle just after the fault occurs before reaching a steady value (see yellow line of $V_{t}$ plot in Fig. 13). The proposed current limitation scheme performs fine, and the current magnitude is largely kept within the 1.414 p.u. limit, as seen in the $I_{\text {mag }}$ plot of Fig. 14. The injected currents, reactive power, and terminal voltages are all restored after the fault is cleared, featuring only limited oscillations in the post-fault currents. The inverter reference voltage does not oscillate and remains within limits, as shown in the $E_{\text {ref }}$ plot in Fig. 14. It is worth noting that the mode remains GF thought the disturbance since the inverter active power output does not hit the MPP limit.

\section{3) Irradiance Change}

In this scenario, the PV system is subject to an irradiance change from 1000 to $500 \mathrm{~W} / \mathrm{m}^{2}$ and back in a few seconds, with the results in Fig. 15 and Fig. 16. This corresponds to very steep irradiance transients of $200 \mathrm{~W} / \mathrm{m}^{2}$ per second, which is among the highest rates recorded and may be caused by moving clouds [39].

In the first few seconds of the irradiance drop, the PV system output is not affected since the irradiance change is reflected only in the reduction of the MPP power and maintained reserves (see $P_{p v}$ in Fig. 16). Once the MPP is reached (vertical red dashed lines in figures), the dc-link voltage falls to the minimum threshold value of $720 \mathrm{~V}$ triggering a mode switch to LGF. In this mode, the frequency adjustment $\Delta \omega$ (Fig. 16) contains the inverter output (yellow line in $P_{i n j}$ plot of Fig. 15) and brings the dc-link voltage back to normal ( $V_{d c}$ plot in Fig. 16). This power deficit is reflected as an under-frequency in the power system, balanced by the remaining generators (Fig. 15).

When the irradiance returns back to normal, the PV-side power increases (see $P_{p v}$ plot in Fig. 16), and the power-sharing among the generators is redistributed (Fig. 15). This change is again reflected in the frequency due to the very steep irradiance rate. When the power to be supplied by the inverter gets back within the capacity of the PV array, $\Delta \omega$ becomes zero, and the mode changes back to normal GF.
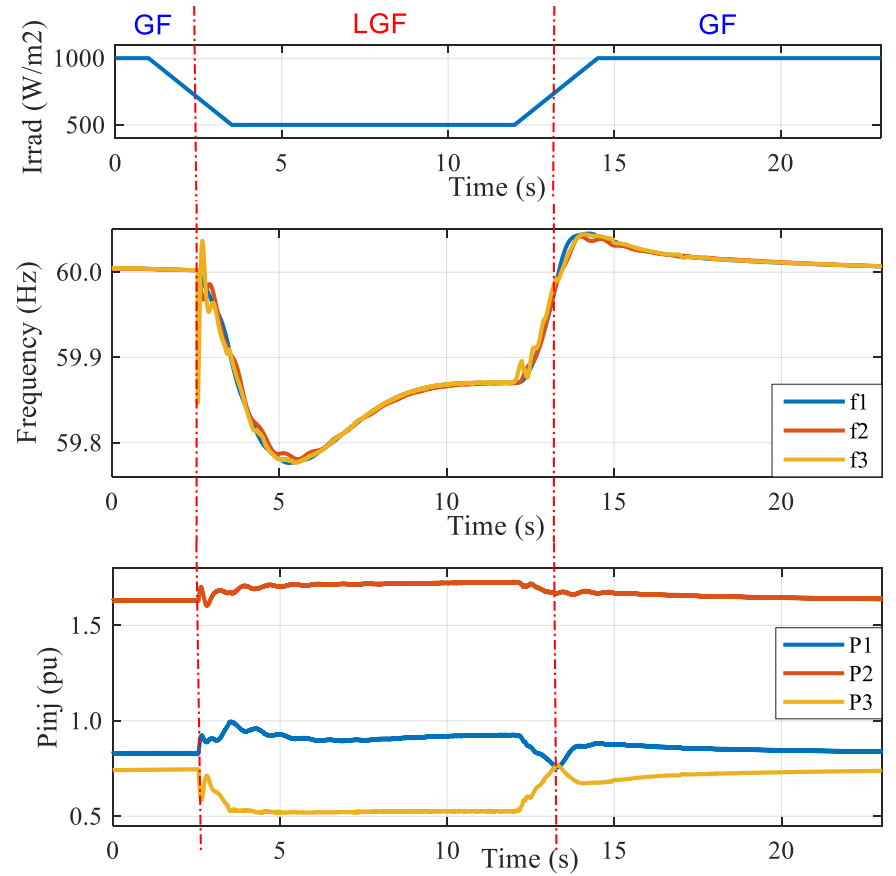

Fig. 15. Frequency and power injection in the SM GFC case subject to irradiance change.

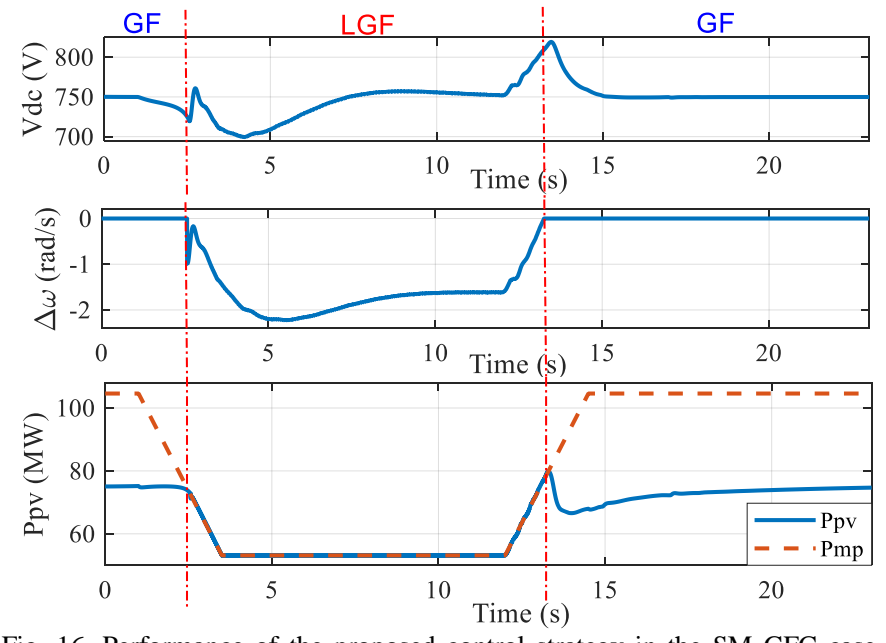

Fig. 16. Performance of the proposed control strategy in the SM GFC case subject to irradiance change.

\section{B. All GFC case (two ideal-input inverters and one PV plant)}

This case study explores the full GFC potential when the entire generation in the power system is inverter-based.

\section{1) Load Disturbance}

The same load disturbance at bus 6 is applied here as well, with the frequency and power injection graphs shown in Fig. 17. Clearly, the frequency drop is substantially smaller, the settling time shorter, and the profile much smoother compared to Fig. 11 of the SM GFC case. This is the outcome of having only fast-acting inverter-based generation with very low droop coefficients $(0.17 \%$ here for all inverters) that can respond promptly to the disturbance and do not suffer from the electromechanical oscillations of the machines, as clearly shown in $P_{i n j}$ plots in Fig. 17.

It is worth noting that such low droop settings are permissible here because all generation is inverter-based; this is in contrast 

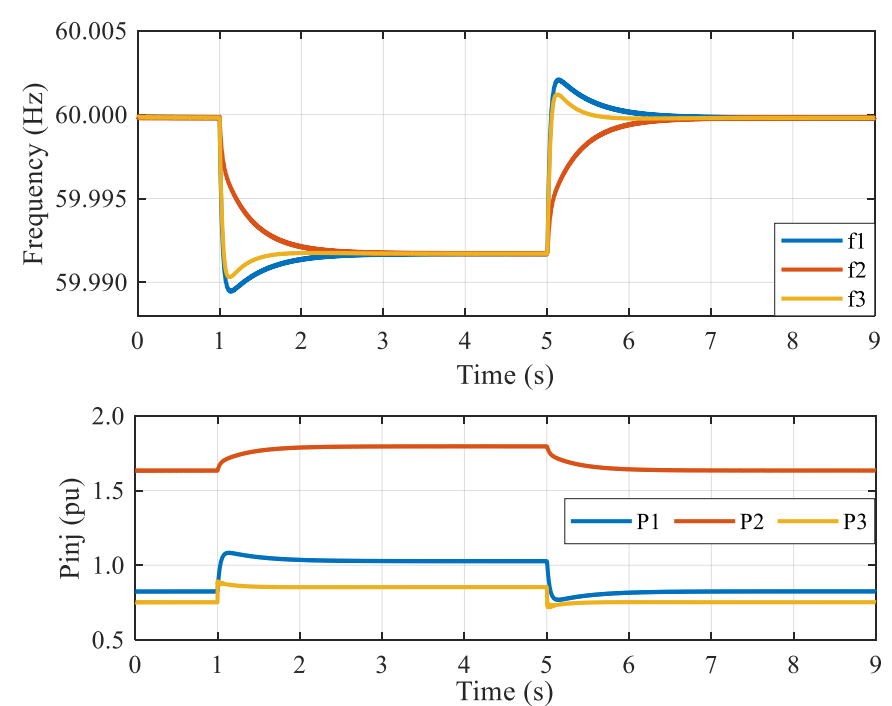

Fig. 17. Frequency and power injection in All GFC case subject to a load change.
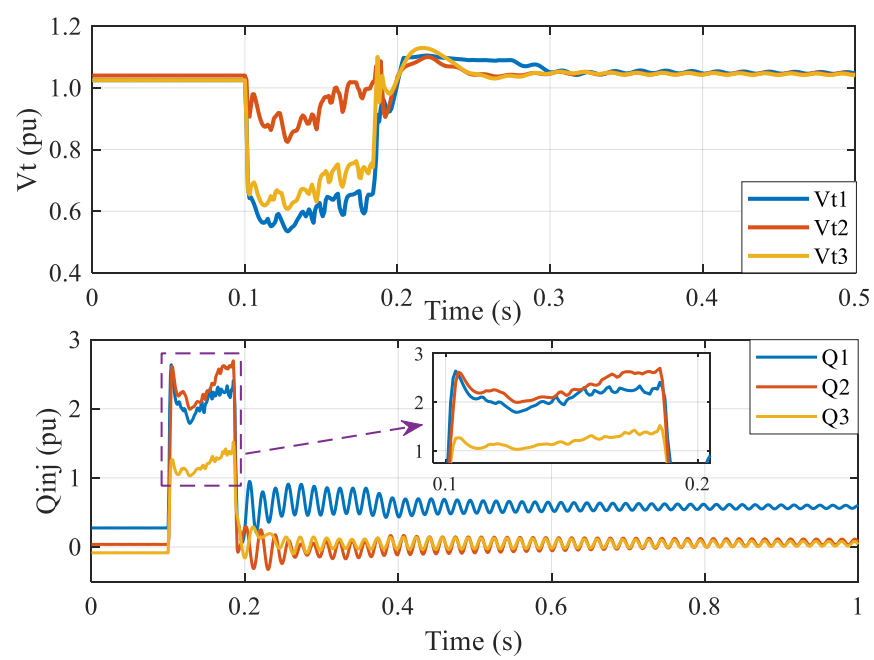

Fig. 18. Voltages and reactive power injections in All GFC case subject to a three-phase fault.

to the previous SM GFC scenario, where the PV plant needs to coordinate with the slow-acting machines and low droop values result in overloading of the PV plant and oscillations in the power system. In the All GFC case, however, neither of the generators is overloaded, as they all equally and quickly share the additional load. This improved load sharing is the reason that the PV GFC plan does not enter in LGF mode here, in contrast to the SM GFC case illustrated in Fig. 12. These results also show that an $100 \%$ inverter-based system with small droop settings does not need any mechanical inertia to operate. This confirms the observation in [42], [8] that GF converters have inherent "electrical inertia" and do not really need emulated or mechanical inertia in the system.

\section{2) Three Phase Fault and Line Opening}

This test case examines the impact of the same fault in line 6-9 when all generation is inverter-based. Here the current limit is set to $k=1.414$ p.u. for all three inverters. The resulting terminal voltage profile and reactive power injections are shown in Fig. 18. The minimum voltage level reached is again about 0.6 p.u., the same as in Fig. 13 of the SM GFC case, but
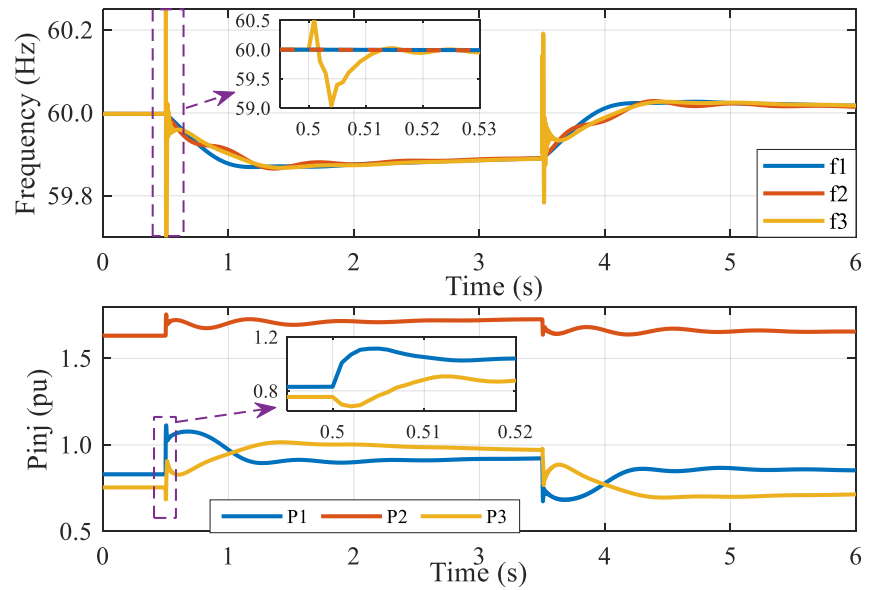

Fig. 19. Frequency and power injections in SM GFL case subject to a load change.

now it is found in node 1 instead of node 3. Apparently, the voltage sag in bus 1 is worse, in bus 2 is much better, and in bus 3 slightly improved compared to the SM GFC case. These changes in the voltage profile are due to different power flows resulting from the inverter's current limitation. This can be easily seen by comparing the reactive power profiles in Fig. 18 and Fig. 13, which exhibit substantially different values during the fault, especially G1 (blue lines). The apparent oscillations in the post-fault reactive power injections are expected, as the fault excites all the system modes and the topology changes due to line tripping. Similar oscillations are present in the case of SMs as well (see $Q_{i n j}$ plot in Fig.13). However, it is worth noting that these oscillations are properly damped within approximately one second after the disturbance (see Fig. 18).

\section{SM GFL case (two SMs and one GFL plant)}

Here, the load disturbance is examined assuming that the PV system runs in the conventional GFL mode with frequency support function [29], [43], rather than in GFC mode. The droop coefficient is $1 \%$, as in the SM GFC case.

The same load disturbance (15\% of the system load) is applied, albeit at different times, with the resulting frequency and active power plots given in Fig. 19. Under such severe disturbances, the PLL may malfunction and extract a distorted frequency for the PV system, as depicted in the frequency plot of Fig. 19. As a result, the power injection of the PV system is not as it should, here featuring a delay and a power dip at the onset of the disturbance (see zoom-box at the power plot in Fig. 19). Such peculiarities are known to be directly related to the PLL, which introduces measurement delays and is prone to errors during severe disturbances. Here this malfunction is not escalated further, but generally such issues become more prevalent at weak and low-inertia grids, as further explored in the following section.

\section{GFC GFL case (one PV GFC and two GFL plants)}

In this case, two GFL inverters with frequency support [29], [43] are connected to bus 1 and 2, and a PV GFC to bus 3. The droop coefficient of the PV GFC remains at $1 \%$, but it is increased to $2 \%$ for the GFL inverters, since lower values result in instability.

The same load disturbance yields the plots of Fig. 20. Like the SM GFL case, the poor PLL performance during the load 

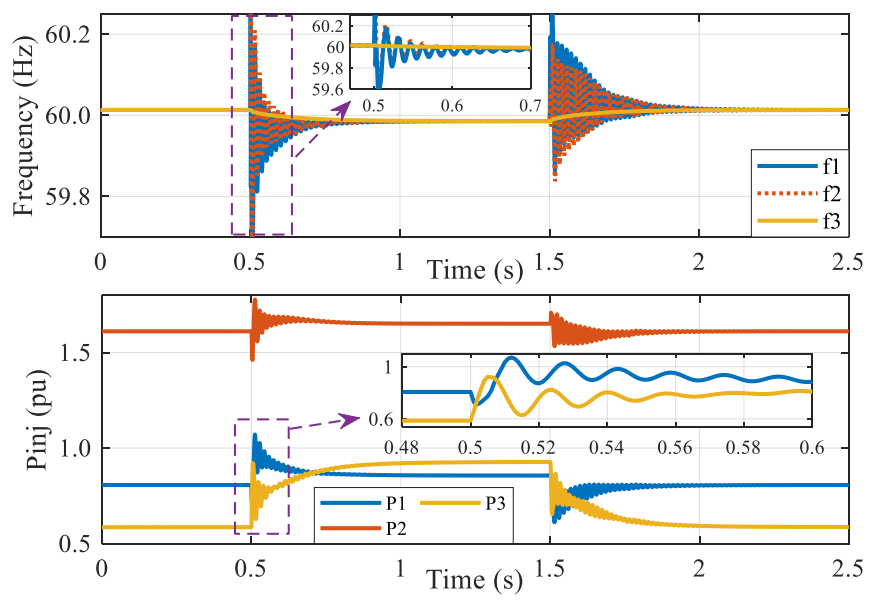

Fig. 20. Frequency and power injection in GFC GFL case subject to a load change.

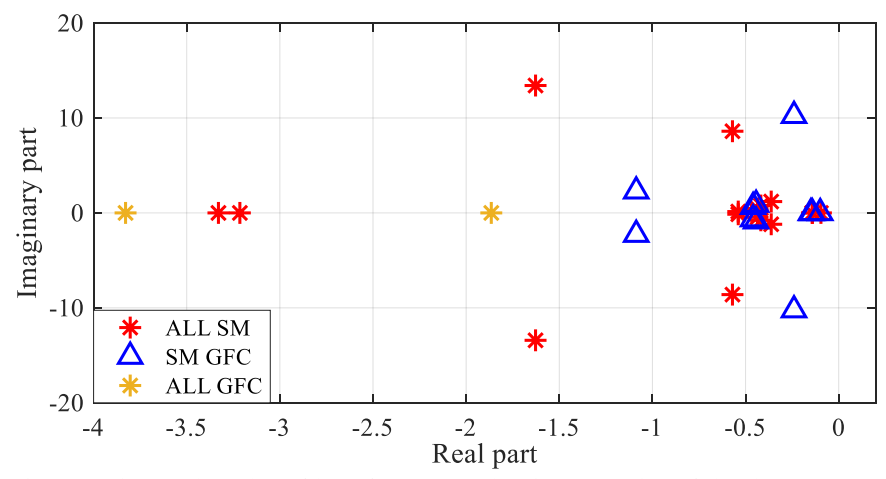

Fig. 21. Eigenvalue plot of low-frequency dominant modes of the three test cases.

switching is reflected in the power injection of the two GFL resources. Here these oscillations are clearly more severe due to the reduced grid strength and lower system inertia, as compared to the previous SM GFL case. It is worth comparing these results to Fig. 17 of the ALL GFC case to observe how different the dynamics are even though both systems are purely converter-based. The main conclusion from this investigation is that a GFC resource is superior to a GFL in terms of stability, and that there should be at least a few of the former in inverterdominated power systems. The identification of the right mixture is not straightforward and is left as future work.

\section{E. Comparison of the All Test Cases}

This section performs a comparative assessment on all five test cases (All SM, SM GFC, All GFC, SM GFL and GFC GFL) by means of modal analysis and dynamic response to a load increment and a three-phase fault.

\section{1) Modal Analysis}

The modal analysis is carried out using fundamental frequency equivalent RMS models of the test cases as a complement to the EMT simulation results. Modal analysis is generally carried out to study the dynamic interactions in the power system; here, it is employed to assess how the smallsignal stability of the power system is affected by replacing synchronous machines with GFC inverters.

The low-frequency dominant eigenvalues of the three main cases (All SM, SM GFC, and All GFC) are shown in Fig. 21. Displacing one SM by an inverter operating in GFC mode

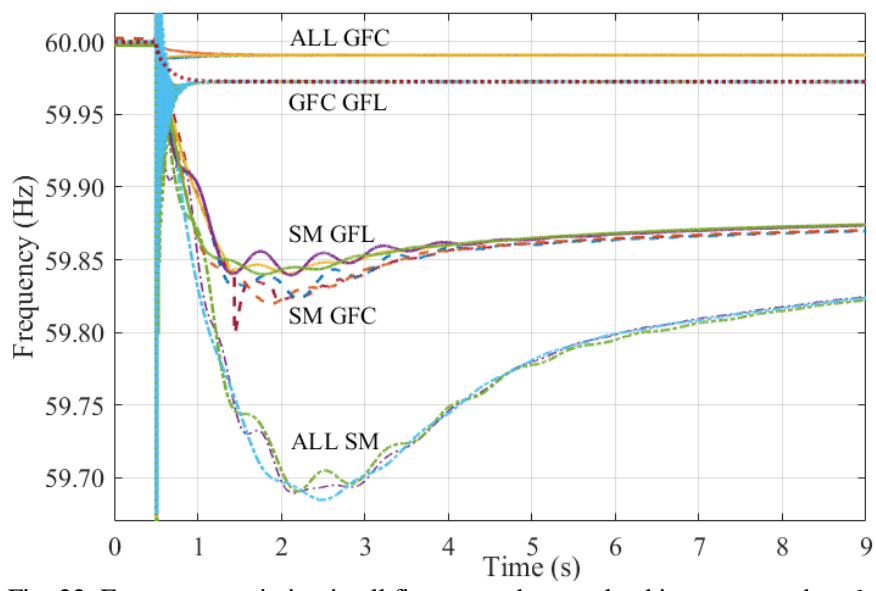

Fig. 22. Frequency variation in all five cases due to a load increment at bus 6 .

affects the low-frequency dominant modes only slightly (red star and blue circle markers in Fig. 21). These low-frequency modes are the root of oscillations found in Fig. 11 and 13 in the previous sections, as well as in the frequency plot of Fig. 22 in the following paragraph. On the contrary, the All GFC case does not feature any oscillatory low-frequency modes; hence the frequency profile during load changes is much smoother and non-oscillatory, as shown in Fig. 17 and Fig. 22. These results show that substituting SMs with GFC inverters neither improves nor worsens the small-signal stability of the system, except when all generation becomes inverter-based, which then exhibits substantially superior performance.

\section{2) Load disturbance}

The big picture on the frequency variation in all five cases for the same load increment (47.25 MW) is shown in Fig. 22. The inverters allow for lower droop settings and respond much faster to the load disturbance than the SMs irrespective of the mode of operation, i.e., either GFL or GFC. Therefore, the frequency profile is better in all inverter cases (All GFC, GFC GFL) as compared to the combination of two SMs and an inverter (SM GFL, SM GFC), the latter being in turn superior to All SM. The SM GFL and SM GFC cases are somewhat similar, being governed mainly by the two SMs inertia and electromechanical dynamics. There is only a PLL-induced frequency spike at the onset of the disturbance in the SM GFL, as discussed in Section V.C.

Between the two purely inverter-based systems, the GFC GFL case exhibits noteworthy post-disturbance oscillations induced by the PLL, which may pose a stability risk and do not allow for very small droop coefficients. This is why the nadir and settling frequency is lower than in ALL GFC that permits even smaller droop coefficients and yields smoother frequency profile. In fact, the main conclusion from this investigation is that replacing SMs with GFL inverters has an upper limit; for higher inverter penetration, they should operate in GFC mode instead. The right balance between GFC and GFL resources remains to this day an open question in the literature [8].

\section{3) Fault}

The terminal voltage of bus 3 in all three cases during a threephase fault followed by line tripping is shown in Fig. 23. Bus 3 is of particular interest, as it is the connection point of the PV system. In general, the terminal voltage is similar during the fault in the three cases, with the lowest value of approximately 


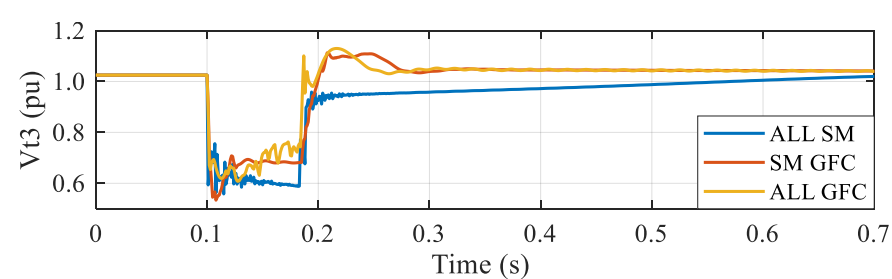

Fig. 23. Bus 3 terminal voltages of all three cases subject to a three-phase fault followed by line tripping.

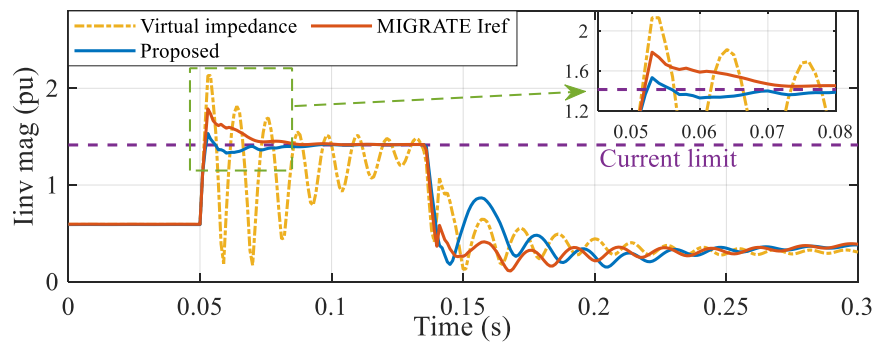

Fig. 24. Comparison of different current limitation schemes.

0.6 p.u. However, the post-fault voltage recovery is faster, although a bit underdamped, when a PV plant is connected to bus 3 in place of an SM (yellow and red lines). These findings show once again that replacing SMs with GFC inverters does not impede the system dynamics during faults, despite the very limited overcurrent capacity of inverters.

\section{F. Comparison of Current Limitation Schemes}

Two widely used current limitation methods [31]-[33] are compared with the proposed method, namely the virtual impedance method from [32] and an implementation of the reference current saturation method based on [31], referred here

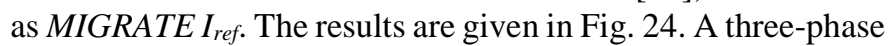
to ground fault occurs at $\mathrm{t}=0.05 \mathrm{sec}$ and is cleared after five cycles. The virtual impedance-based method suffers from the initial current overshoot and an oscillatory response during the fault. The MIGRATE $I_{\text {ref }}$ method also results in an initial nonoscillatory overcurrent. On the contrary, the proposed method successfully suppresses the initial overshoot thanks to the rate limiters and adjustable saturation limits, which also do not allow for any oscillations in the injected current. This case study confirms the superior performance of the proposed method as opposed to other standard approaches and validates the modifications adopted in the reference current limitation scheme.

\section{CONCLUSIONS}

This paper introduces a new GFC scheme for PV systems that do not employ real-time estimation of the MPP and make optimal use of the limited power reserves. By operating in full or limited grid-forming mode, the PV plant preserves its voltage source nature and manages to assist the grid during disturbances similarly or even better than synchronous machines. The modified current saturation scheme performs smoothly, without any need for fault detection or control switching.

Replacing SMs with PV GFC results in improved frequency profile during load disturbances due to faster response from the PV plant, and comparable terminal voltage profiles during faults despite the strict inverter overcurrent limits. However, the
PV GFC introduces another source of disturbances to the power system resulting from irradiance transients during cloud movement.

Inverters in GFL mode with ancillary services can support the grid during disturbances, but the contribution becomes limited as the system strength decreases. The GFC mode of inverter operation is the way forward for the renewables-rich and inverter-dominated power systems of the future.

Future work involves a complete investigation of the dynamic interactions between GFC and GFL inverters and the rest of the power system at various sizes and generation mixtures. Similarly, a methodology to determine the appropriate ratio of GFC and GFL resources would be very useful in converter-dominated power systems. Furthermore, the proposed method is designed for uniform illumination, which is the common assumption for utility-scale PV systems; an extension of the method to partial shading would improve its credibility and reliability at all possible conditions.

\begin{tabular}{l} 
APPENDIX \\
\multicolumn{1}{|c|}{ TABLE I. PV SYSTEM PARAMETERS } \\
\begin{tabular}{|l|l|}
\hline PV plant & $105 \mathrm{MWp} @ 1000 \mathrm{~W} / \mathrm{m}^{2}$ and $25^{\circ} \mathrm{C}$, \\
& $p^{*}=75 \mathrm{MW}, P_{\text {res }}=30 \mathrm{MW}$ \\
\hline Inverter & $S=25 \mathrm{kVA}, f_{s w}=10 \mathrm{kHz}, K_{p i}=14.85$, \\
& $K_{i i}=1632, K_{p v}=0.0142, K_{i v}=4.17$ \\
\hline Grid-forming & $d_{\omega}=1 \%, \omega_{c}=2 \pi^{*} 10 \mathrm{rad} / \mathrm{sec}$ \\
\hline Closed-loop voltage control & $K_{p}=0.2, K_{i}=1$ \\
\hline$\Delta \omega$ PI controller & $K_{p}=1.5, K_{i}=3$ \\
\hline DC-DC conv. controller & $K_{p}=0.1, K_{i}=2$ \\
\hline Current limitation & $k=1.414, k_{l}=1.2$ \\
\hline
\end{tabular}
\end{tabular}

\section{REFERENCES}

[1] F. Milano, F. Dörfler, G. Hug, D. J. Hill, and G. Verbič, "Foundations and challenges of low-inertia systems (Invited Paper)," Power Syst. Comp. Conf. (PSCC), Dublin, Ireland, 2018.

[2] C. Loutan, P. Klauer, S. Chowdhury, S. Hall, M. Morjaria, V. Chadliev, N. Milam, C. Milan, and V. Gevorgian, "Demonstration of essential reliability services by a 300-MW solar photovoltaic power plant," National Renewable Energy Lab. (NREL), Golden, CO, United States, Rep. NREL/TP-5D00-67799, 2017.

[3] ENTSO-E, "Need for synthetic inertia (SI) for frequency regulation: ENTSO-E guidance document for national implementation for network codes on grid connection," ENTSO-E, Brussels, Belgium, Tech. Guideline, Jan. 2018.

[4] J. C. Hernandez, P. G. Bueno, and F. Sanchez-Sutil, "Enhanced utilityscale photovoltaic units with frequency support functions and dynamic grid support for transmission systems," IET Ren. Power Gen., vol. 11, no. 3, pp. 361-372, Jan. 2017.

[5] C. Guo, S. Yang, W. Liu, C. Zhao, and J. Hu, "Small-signal stability enhancement approach for VSC-HVDC system under weak AC grid conditions based on single-input single-output transfer function model," IEEE Trans. Power Del., to be published. DOI: 10.1109/TPWRD.2020.3006485.

[6] S. Lu, Z. Xu, L. Xiao, W. Jiang, and X. Bie, "Evaluation and enhancement of control strategies for VSC stations under weak grid strengths," IEEE Trans. Power Syst., vol. 33, no. 2, pp. 1836-1847, Jun. 2017.

[7] D. Sun, H. Liu, S. Gao, L. Wu, P. Song, and X. Wang, "Comparison of different virtual inertia control methods for inverter-based generators," J. Mod. Power Syst. Clean Energy, vol. 8, no. 4, pp. 768777, Jun. 2020.

[8] Y. Lin, J. H. Eto, B. B. Johnson, J. D. Flicker, R. H. Lasseter, H. N. Villegas Pico, G. S. Seo, B. J. Pierre, and A. Ellis, "Research roadmap on grid-forming inverters," Tech. Rep. No. NREL/TP-5D00-73476, National Renewable Energy Lab (NREL), Golden, CO (U. S.), 2020. 
[9] E. I. Batzelis, et al., "Solar integration in the UK and India: technical barriers and future directions," JUICE white paper on solar integration, 2020.

[10] B. Kroposki, B. Johnson, Y. Zhang, V. Gevorgian, P. Denholm, B. M. Hodge, and B. Hannegan, "Achieving a 100\% renewable grid: Operating electric power systems with extremely high levels of renewable energy," IEEE Power Energy Mag., vol.15, no. 2, pp.61-73, Mar. 2017.

[11] ENTSO-E, "High Penetration of Power Electronic Interfaced Power Sources (HPoPEIPS): ENTSO-E Guidance document for national implementation for network codes on grid connection," ENTSO-E, Brussels, Belgium, Tech. Guideline, Mar. 2017.

[12] D. Pattabiraman, R. H. Lasseter, and T. M. Jahns, "Comparison of grid following and grid forming control for a high inverter penetration power system," IEEE PES GM 2018, Portland, OR, USA, 2018.

[13] A. Tayyebi, D. Groß, A. Anta, F. Kupzog, and F. Dörfler, "Frequency stability of synchronous machines and grid-forming power converters," IEEE Trans. Emerg. Sel. Topics Power Electron., vol. 8, no. 2, pp. 10041018, Jan. 2020.

[14] D. Pattabiraman, R. H. Lasseter, and T. M. Jahns, "Short-term voltage stability of power systems with high inverter penetration under small disturbances," IEEE PES GM 2019, Atlanta, GA, USA, 2019.

[15] M. C. Chandorkar, D. M. Divan, and R. Adapa, "Control of parallelconnected inverters in standalone AC supply systems," IEEE Trans. Ind. Appl., vol. 29, no. 1, pp. 136-143, Jan. 1993.

[16] N. Pogaku, M. Prodanovic, and T. C. Green, "Modeling, analysis, and testing of autonomous operation of an inverter-based microgrid," IEEE Trans. Power Electron., vol. 22, no. 2, pp. 613-625, Mar. 2007.

[17] A. Tayyebi, F. Dörfler, F. Kupzog, Z. Miletic, and W. Hribernik, "Gridforming converters-inevitability, control strategies and challenges in future grids application," Proc. CIRED 2018, Ljubljana, Slovenia, Jun. 2018.

[18] M. Chen, D. Zhou, and F. Blaabjerg, "Modelling, implementation, and assessment of virtual synchronous generator in power systems," $J$. Mod. Power Syst. Clean Energy, vol. 8, no. 3, pp. 399-411, Apr. 2020.

[19] L. Huang, H. Xin, Z. Wang, K. Wu, H. Wang, J. Hu, and C. Lu, "A virtual synchronous control for voltage-source converters utilizing dynamics of DC-link capacitor to realize self-synchronization," IEEE Trans. Emerg. Sel. Topics Power Electron., vol. 5, no. 4, pp. 1565-1577, Aug. 2017.

[20] M. Colombino, D. Groß, J. S. Brouillon, and F. Dörfler, "Global phase and magnitude synchronization of coupled oscillators with application to the control of grid-forming power inverters," IEEE Trans. Autom. Control, vol. 64, no. 11, pp. 4496-511, Feb. 2019.

[21] M. E. Elkhatib, W. Du, and R. H. Lasseter, "Evaluation of inverter-based grid frequency support using frequency-watt and grid-forming PV inverters," IEEE PES GM 2018, Portland, OR, USA, 2018.

[22] Z. Chen, R. H. Lasseter, and T. M. Jahns, "Power reserve for gridforming PV sources with stability enhancement in mixed-source microgrids," IEEE PES GM 2019, Atlanta, GA, Aug. 2019.

[23] Z. Chen, R. H. Lasseter, and T. M. Jahns, "Active power reserve control for grid-forming PV sources in microgrids using model-based maximum power point estimation," Proc. IEEE ECCE, Oct. 2019, pp. 41-48.

[24] R. H. Lasseter, Z. Chen, and D. Pattabiraman, "Grid-forming inverters: A critical asset for the power grid," IEEE Trans. Emerg. Sel. Topics Power Electron., vol.8, no.2, pp.925-935, Dec. 2019.

[25] Z. Chen, R. H. Lasseter, and T. M. Jahns, "Overcurrent limiting and dc bus voltage ripple minimization in grid-forming PV sources under grid voltage sags," Proc. IEEE PEDG, Xi'an, China, 2019, pp.964-971.

[26] V. Gevorgian, Highly accurate method for real-time active power reserve estimation for utility-scale photovoltaic power plants, NREL Tech. Report No. NREL/TP-5D00-73207, National Renewable Energy Lab. (NREL), Golden, CO (United States), Feb. 2019.

[27] E. Batzelis, A. Junyent-Ferre, and B. C. Pal, "MPP estimation of PV systems keeping power reserves under fast irradiance changes," in Proc. IEEE PES GM 2020, Montreal, QC, Canada, Aug. 2020.

[28] E. I. Batzelis, S. A. Papathanassiou, and B. C. Pal, "PV system control to provide active power reserves under partial shading conditions, "IEEE Trans. Power Electron., vol. 31, no. 11, pp. 9163-9175, Nov. 2018.

[29] E. I. Batzelis, G. Anagnostou, I. R. Cole, T. R. Betts, and B. C. Pal, "A state-space dynamic model for photovoltaic systems with full ancillary services support," IEEE Trans. Sust. Energy, vol. 10, no. 3, pp. pp. 13991409, Nov. 2018.

[30] E. I. Batzelis, G. E. Kampitsis, and S. A. Papathanassiou, "Power reserves control for PV systems with real-time MPP estimation via curve fitting," IEEE Trans. Sust. Energy, vol. 8, no. 3, pp. 1269-1280, Feb. 2017.

[31] T. Qoria, Q. Cossart, C. Li, X. Guillaud, F. Colas, F. Gruson, and X. Kestelyn, "Deliverable 3. 2: local control and simulation tools for large transmission systems," MIGRATE Project, Tech. Rep., 2018. [Online]. available: https://www.h2020-migrate.eu/downloads.html.

[32] A. D. Paquette and D. M. Divan, "Virtual impedance current limiting for inverters in microgrids with synchronous generators," IEEE Trans. Ind. Appl., vol. 51, no. 2, pp. 1630-1638, Aug. 2014.

[33] G. Denis, T. Prevost, M. S. Debry, F. Xavier, X. Guillaud, and A. Menze, "The Migrate project: the challenges of operating a transmission grid with only inverter-based generation - A grid-forming control improvement with transient current-limiting control," IET Ren. Power Gen., vol. 12, no. 5, pp. 523-529, Mar. 2018.

[34] L. Zhang, L. Harnefors, and H. P. Nee, "Power-synchronization control of grid-connected voltage-source converters," IEEE Trans. Power Syst., vol. 25, no. 2, pp. 809-820, Nov. 2009.

[35] J. Wang, A. Pratt, and M. Baggu, "Integrated synchronization control of grid-forming inverters for smooth microgrid transition," IEEE PES GM 2019, Atlanta, GA, USA, 2019.

[36] T. Esram, and P. L. Chapman, "Comparison of photovoltaic array maximum power point tracking techniques," IEEE Trans. Energy Conv., vol. 22, no. 2, pp. 439-449, June 2007.

[37] P.W. Sauer, M.A. Pai, and J. H. Chow, Power System Dynamics and Stability with Synchrophasor Measurement and Power System Toolbox. $2^{\text {nd }}$ ed., Hoboken, NJ: Wiley IEEE Press, 2018, pp. 142-145.

[38] RCSAD Manual: Benchmark Power System Cases, $5^{\text {th }}$ ed., RTDS Technologies, Winnipeg, Manitoba, Canada, 2016.

[39] V. Purba, B. B. Johnson, S. Jafarpour, F. Bullo, and S. V. Dhople, "Dynamic aggregation of grid-tied three-phase inverters," IEEE Trans. Power Syst., vol. 35, no. 2, pp. 1520-1530, Sep. 2019.

[40] T. Qoria, F. Gruson, F. Colas, X. Guillaud, M-S Debry, and T. Prevost, "Tuning of cascaded controllers for robust grid-forming voltage source converter," Power Syst. Comp. Conf. (PSCC), Dublin, Ireland, 2018.

[41] W. Xiao, W. G. Dunford, P. R. Palmer, and A. Capel, "Regulation of photovoltaic voltage," IEEE Trans. Ind. Electron., vol. 54, no. 3, pp. 1365-1374, July 2007.

[42] T. Prevost and G. Denis, "Deliverable 3.6: Requirement guidelines for operating a grid with $100 \%$ power electronic devices," MIGRATE Project, Tech. Rep., 2019. [Online]. available: https://www.h2020migrate.eu/downloads.html.

[43] J. Johnson, J. C. Neely, J. Delhotal, and M. Lave, "Photovoltaic frequency-watt curve design for frequency regulation and fast contingency reserves," IEEE Jour. Photovoltaics, vol. 6, no. 6, pp. 1611-1618, 2016.

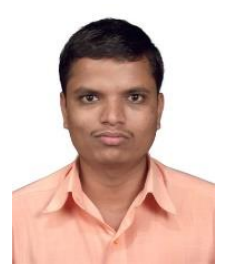

Bandopant Pawar (S'15) received the M.Tech. degree from Indian Institute of Technology Roorkee, Roorkee, India in 2015. Currently, he is pursuing his $\mathrm{Ph} . \mathrm{D}$. from Indian Institute of Technology Kanpur, Kanpur, India. His research interests include grid integration of renewable energy sources, power system dynamics, stability and control of power systems with large penetration of renewable sources.

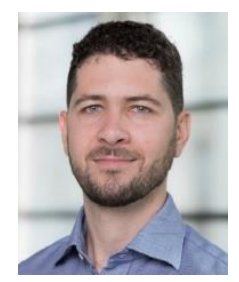

Efstratios I. Batzelis (S'14-M'17-SM'19) has been a Lecturer at the University of Southampton, Southampton, UK since April 2021 and a Research Fellow of the Royal Academy of Engineering (RAEng) working on solar integration in developing countries. Prior to that, he was a Research Fellow at Imperial College London, London, UK since 2019 and before that he held an EU Marie-Curie individual fellowship on photovoltaic control \& integration in 2017-2019 also at Imperial College London. He obtained his $\mathrm{PhD}$ degree from the National Technical University of Athens (NTUA), Athens, Greece, in 2016. He is currently serving as Associate Editor in 
IEEE Transactions on Sustainable Energy. His research interests involve renewable energy technologies and distributed energy resources, mainly solar photovoltaics, power electronics control and power system dynamics and stability.

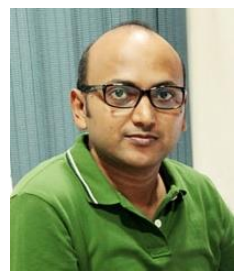

Saikat Chakrabarti (S'06-M'07-SM'11) received the $\mathrm{Ph} . \mathrm{D}$. degree in electrical engineering from Memorial University of Newfoundland, St. Johns, NF, Canada, in 2006. He is currently a Professor with IIT Kanpur, Kanpur, India. His research interests include state estimation of transmission and distribution systems, stability and control of power systems with large penetration of renewable energy, microgrids, modelling of generators and loads, and planning and operation of distribution systems.

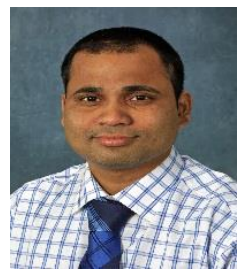

Bikash C. Pal (M'00-SM'02-F'13) received B.E.E. (with honors) degree from Jadavpur University, Calcutta, India, M.E. degree from the Indian Institute of Science, Bangalore, India, and Ph.D. degree from Imperial College London, London, U.K., in 1990, 1992, and 1999, respectively, all in electrical engineering. Currently, he is a Professor in the Department of Electrical and Electronic Engineering, Imperial College London. His current research interests include renewable energy modelling and control, state estimation, and power system dynamics. He is Vice President Publications, IEEE Power \& Energy Society. He was Editor-in-Chief of IEEE Transactions on Sustainable Energy (2012-2017) and Editor-in-Chief of IET Generation, Transmission and Distribution (2005-2012) and is a Fellow of IEEE for his contribution to power system stability and control. 